\title{
EL PRINCIPIO DE INTEGRALIDAD DEL SISTEMA GENERAL DE SEGURIDAD SOCIAL EN SALUD Y LAS ENFERMEDADES CATASTRÓFICAS EN COLOMBIA
}

\section{THE PRINCIPLE OF INTEGRALITY OF THE GENERAL SYSTEM OF SOCIAL SECURITY IN HEALTH AND CATASTROPHIC DISEASES IN COLOMBIA}

\author{
Dra. Luisa Fernanda Martínez Espinosa ${ }^{1}$ \\ Universidad Santiago de Cali \\ https://orcid.org/0000-0003-0366-8164 \\ luisa.martinez00@usc.edu.com \\ Colombia \\ Abogada Alejandra García Fernández $z^{2}$ \\ Universidad Santiago de Cali \\ https://orcid.org/0000-0001-5964-4431 \\ aleja_gf@hotmail.com \\ Colombia
}

\section{SUMARIO}

- Introducción

- Análisis doctrinal de la relación entre el principio de integralidad y las enfermedades catastróficas

- Análisis jurisprudencial de la relación entre el principio de integralidad y las enfermedades catastróficas

- Conclusión

- Bibliografía

\section{RESUMEN}

El propósito de este artículo es analizar el alcance jurídico del principio de integralidad del Sistema General de Seguridad Social en Salud y las enfermedades catastróficas en Colombia tomando como base la doctrina y la jurisprudencia emanada de la Corte Constitucional. Esta investigación tiene un nivel de profundidad descriptivo- explicativo porque se especifica las características y las causas del fenómeno, el método es hermenéutico toda vez que parte de la interpretación para establecer la relación entre las variables objeto de estudio. En la actualidad, las personas que padecen dichas enfermedades se encuentran con muchas barreras para acceder al sistema de salud: falta de información, falta de diagnóstico efectivo y la negación de medicamentos; la materialización del principio de integralidad establecido en el ordenamiento jurídico colombiano garantiza la atención oportuna, eficiente y con calidad.

\begin{abstract}
The purpose of this article is to analyze the legal scope of the principle of comprehensiveness of the General System of Social Security in Health and catastrophic diseases in Colombia based on the doctrine and jurisprudence emanating from the Constitutional Court. This research has a descriptive-explanatory
\end{abstract}

1 Doctora en Derecho con mención de reconocimiento por la Universidad Externado de Colombia. Consejera de la Facultad de Derecho USC. Integrante del Grupo de Investigación en Ciencia Política, Derecho y Relaciones Internacionales Categoría A en Minciencias. Profesora y Directora del Semillero de Investigación Eduardo Umaña Seccional Palmira de la Universidad Santiago de Cali. Correo institucional: luisa.martinez00@usc.edu.co

2 Abogada de la Universidad Santiago de Cali. Especialista en Seguridad Social de la Universidad Javeriana. Integrante del Semillero de Investigación Eduardo Umaña Seccional Palmira de la Universidad Santiago de Cali, adscrito al Grupo de Investigación en Ciencia Politica, Derecho y Relaciones Internacionales Categoría A en Minciencias. Correo electrónico: aleja_gf@hotmail.com 
level of depth because the characteristics and causes of the phenomenon are specified, the method is hermeneutical since it starts from the interpretation to establish the relationship between the variables under study. Currently, people with various diseases face many barriers to accessing the health system: lack of information, lack of effective diagnosis and denial of medications; The materialization of the principle of comprehensiveness established in the Colombian legal system guarantees timely, efficient and quality care.

\section{PALABRAS CLAVE}

Enfermedades catastróficas; Eps; jurisprudencia; principio de integralidad; seguridad social; salud

\section{KEYWORDS}

Catastrophic illnesses; Eps; jurisprudence; integrality principle; social security; health

\section{INTRODUCCIÓN}

Uno de los principios que rige el Sistema de Seguridad Social en Salud es el principio de integralidad, el cual ha sido definido en el artículo 2 de la Ley 100 de 1993 como "la cobertura de todas las contingencias que afectan la salud, la capacidad económica y en general las condiciones de vida de toda la población...". Si bien, este principio brinda una protección para las contingencias consagradas en la Ley 100 de 1993, se puede suscitar una serie de exclusiones para brindar una atención integral a aquellas personas que padecen alguna enfermedad catastrófica, puesto que estas enfermedades necesitan que se garantice el servicio a la salud de manera completa desde la prevención hasta llegar a la rehabilitación. Estas enfermedades se encuentran definidas en el artículo 16 de la Resolución 5261 de 1994 "Para efectos del presente decreto se definen como enfermedades ruinosas o catastróficas, aquellas que representan una alta complejidad técnica en su manejo, alto costo, baja ocurrencia y bajo costo efectividad en su tratamiento".

El principio ha sido desarrollado por la Corte Constitucional en varios pronunciamientos, entre ellos se encuentra la sentencia T-418 del 2013, donde se señaló que los servicios de salud y tratamientos que se le brindan a las personas debe ser integral para así no poner en riesgo los derechos fundamentales, de manera que la integralidad comprende lo siguiente:

«un conjunto de cuidado, suministro de medicamentos, intervenciones quirúrgicas, prácticas de rehabilitación, exámenes para el diagnóstico y el seguimiento, así como todo otro componente que el médico tratante valore como necesario para el pleno restablecimiento de la salud del paciente o para mitigar las dolencias que le impiden llevar su vida en mejores condiciones; y en tal dimensión, debe ser proporcionado a sus afiliados por las entidades encargadas de prestar el servicio público de la seguridad social en salud» (párr.75)

En la Sentencia T-002 de 2016, se señala que el tratamiento que se debe adelantar no tiene como finalidad la curación, sino que debe de estar dirigido a superar todas las calamidades que pongan en peligro la vida, la dignidad y la integridad de las personas, la enfermedad se debe tratar desde un enfoque integral, que comprenda todos los procedimientos fundamentales para el mejoramiento social, mental y funcional del paciente. Sin embargo, hay ocasiones en que no se logra una rehabilitación, pero esto no es obstáculo para brindar por todos los medios mejorar la calidad de vida del paciente a través de los diferentes medicamentos y seguimientos.

En el sistema de salud colombiano los planes que ofrecen las EPS excluyen muchos medicamentos, tratamientos y procedimientos generando con ello una barrera de acceso a la salud para toda la población, incluyendo personas con especial protección, se requiere que las enfermedades catastróficas tengan una mayor cobertura, y esto se puede realizar a partir de la interpretación del principio de integralidad, que cubre todas las contingencias que afectan a la salud. En tal sentido esta investigación entraña una relevancia jurídica y social porque se analiza desde la doctrina y la jurisprudencia el principio de integralidad del Sistema General de Seguridad Social en Salud y su proyección en las enfermedades catastróficas. 
salud y las enfermedades catastróficas en colombia

The principle of integrality of the general system of social security

in health and catastrophic diseases in colombia

\section{ANÁLISIS DOCTRINAL DE LA RELACIÓN ENTRE EL PRINCIPIO DE INTEGRALIDAD Y LAS ENFERMEDADES CATASTRÓFICAS}

El origen de la Seguridad Social se remonta a doctrinas políticas y sociales, es así como surge en Europa más específicamente en Alemania la Seguridad social como mecanismo para mejorar las condiciones sociales y económicas de los trabajadores de las empresas. En el mundo moderno, la seguridad social, surge como un instrumento de protección al hombre contra determinadas necesidades sociales, ya que el ser humano intenta satisfacerlas acudiendo a su propio esfuerzo, mediante un trabajo que le ayude a recaudar los recursos necesarios, y también requiere de la capacidad de previsión y de la solidaridad humana, ya que a través de ello se ofrece mecanismos adicionales para atender las necesidades sociales (Arenas, 2008).

En Colombia, el sistema de seguridad social surgió en la época de 1945 y 1946 donde se originó la Caja Nacional de Previsión (CAJANAL) y el Instituto Colombiano de los Seguros Sociales (ISS), antes de la existencia de dichas entidades, los trabajadores contaban con algunas prestaciones como la salud, cesantías, riesgos profesionales y pensiones, denominadas prestaciones patronales, con el tiempo, las empresas se vieron en la imposibilidad de pagar aquellas prestaciones, generando así la exigencia de crear un sistema de seguridad social alejado de las empresas, para que se ocupara de todos los derechos de los trabajadores. Es así como la seguridad social surgió como un mecanismo institucional y financiero que aseguraba que los trabajadores pudieran acceder a sus prestaciones sociales (Rocha, 2010).

El Sistema General de Seguridad Social en Salud tiene entre sus finalidades garantizar el derecho fundamental a la salud bajo el principio de integralidad el cual consiste en prestar el servicio en condiciones de "continuidad, oportunidad, disponibilidad y accesibilidad", de esta forma, el Plan Obligatorio de Salud, considerado como procedimientos, insumos $\mathrm{y}$ medicamentos tienen una labor muy importante para el fortalecimiento del principio de integralidad, y de este modo es fundamental para garantizar el derecho a la salud a las personas que son sujetos de especial protección, como son aquellas que padecen enfermedades catastróficas. Aunque existe normatividad que regula estas enfermedades, existen barreras y falencias administrativas dentro del Sistema que impide el cumplimiento del principio de integralidad en las personas que padecen dichas enfermedades (Garzón, 2014)

El principio de integralidad, también se encuentra definido en el artículo 8 de la Ley 1751 del 2015 como "los servicios y tecnologías de salud deberán ser suministrados de manera completa para prevenir, paliar o curar la enfermedad, con independencia del origen de la enfermedad o condición de salud, del sistema de provisión, cubrimiento o financiación definido por el legislador...". Asimismo, en el artículo 15 de la misma ley, se encuentra una serie de servicios excluidos cuando:
a) tengan como finalidad principal un propósito cosmético o suntuario no relacionado con la recuperación o mantenimiento de la capacidad funcional o vital de las personas; b) Que no exista evidencia científica sobre su seguridad y eficacia clínica; c) Que no exista evidencia científica sobre su efectividad clínica; d) Que su uso no haya sido autorizado por la autoridad competente; e) Que se encuentren en fase de experimentación; f) Que tengan que ser prestados en el exterior.

Frente a estas exclusiones, mediante la Sentencia C-313 del 2014, se ha dicho que sí se puede garantizar estas exclusiones siempre que se cumplan unos requisitos consagrados en el numeral 5.2.15.3 de la respectiva sentencia, entre los que se encuentra cuando el medicamento o procedimiento sea necesario para garantizar el derecho a la vida e integridad del paciente, que no exista otro medicamento que proteja la salud del paciente, que el paciente carezca de capacidad económica para sufragar el fármaco o el procedimiento requerido, o que el medicamento o procedimiento sea ordenado por su médico tratante.

Ante este pronunciamiento, es evidente que el servicio de salud no se debe de brindar de manera fraccionada, y se debe de garantizar un tratamiento completo así se encuentre dentro de las exclusiones, pero cumplan con los requisitos mencionados anteriormente.

Por otro lado, este principio no sólo se refiere a la prestación de los servicios de salud, sino 
que este se dé en condiciones de oportunidad y eficiencia, pero existen situaciones en donde las personas que padecen enfermedades catastróficas como los enfermos de VIH requieren mayor atención sobrepasando las coberturas del POS, generando con ello que sus necesidades no sean cubiertas por el sistema de salud.

Las prestaciones consagradas en los "planes de salud" no garantizan una prestación integral, ya que existe exclusión de algunos medicamentos, procedimientos e insumos que impiden la prestación de manera completa de los servicios de salud. Es por ello que, para garantizar la integralidad en la salud, es importante considerar la salud como un derecho fundamental para que a través de la normatividad se incluyan los servicios que sean necesarios para proteger a las personas que padecen enfermedades catastróficas sin que tengan que instaurar acciones de tutela para exigir su cumplimiento.

El VIH, es considerado como una enfermedad catastrófica la cual se encuentra regulada en el Decreto 1543 de 1997 en donde se establece los términos bajo los cuales se debe entender el VIH y el SIDA, además de las medidas que se deben tomar para garantizar su protección integral, es decir que "el VIH es el retrovirus agente causal del SIDA y éste a su vez es el conjunto de síntomas y signos generados por el compromiso del sistema inmunológico de una persona como consecuencia de la infección por el VIH" (p.13-14). Este decreto consagra la integralidad como elemento fundamental para la atención de esta enfermedad, en donde a través de este principio de debe brindar atención en las áreas de "prevención, diagnóstico, tratamiento y rehabilitación" que se requiera para combatir la enfermedad, además de mejorar la calidad de vida del paciente.

La Ley 972 de 2005, regula todo lo concerniente a las enfermedades catastróficas y ruinosas, sin embargo, hace mayor énfasis a la enfermedad VIH, además establece las sanciones ante la negación de la atención y consagra las medidas necesarias para disminuir los costos de los medicamentos que se necesita para dicha enfermedad, sin embargo, esta ley no establece medidas integrales para su prevención.

\section{Perspectivas del principio de integralidad}

Como se mencionó inicialmente, si bien el principio de integralidad, se encuentra definido en la Ley 100 de 1993, la Corte Constitucional ha dado mayor relevancia y ha estructurado la integralidad bajo 3 perspectivas "(i) como una forma de garantizar el derecho a la salud (ii) como una regla de prestación del servicio de salud (iii) como una característica del sistema de salud y (iv) como una obligación acerca de las condiciones como debe prestarse el servicio" (Garzón, 2014, p.21).

\section{(i) El principio de integralidad como una forma de garantizar el derecho a la salud (p.21)}

En la sentencia T-1041 de 2012, se hace referencia al principio de integralidad como aquel mediante el cual se puede llegar a obtener el más alto nivel de salud, por ende, menciona la sentencia T-760 de 2008, la cual consagra que para el cumplimiento de este principio se necesita el suministro de medicamentos, exámenes, diagnósticos en pro de la salud del paciente, conforme lo ordenado por su médico tratante. Es decir, que el objetivo del principio de integralidad es suministrar integralmente todas las atenciones que se requiera para mitigar las dolencias, además que a través de este principio se puede retrasar el deterioro de la salud para las personas que padecen enfermedades catastróficas.

\section{(ii) El principio de integralidad como regla del servicio (p.21)}

A través de la sentencia C-626 de 2009, se manifestó que la integralidad se debe de analizar desde el área de la "educación, la información, el diagnóstico, el tratamiento y la rehabilitación otorgados según la intensidad de uso y los niveles de atención en condiciones de cantidad, oportunidad, calidad y eficiencia." (p.21). De esta forma, la Corte ha manifestado que la garantía del derecho a la salud sólo se puede dar cuando se protegen en totalidad las necesidades del bienestar humano, de esta manera, por ejemplo, los tratamientos que requieran los pacientes de $\mathrm{VIH}$ a pesar que se encuentren dentro del POS, es importante revisar el contexto y la manera en que se brindan, 
salud y las enfermedades catastróficas en colombia

The principle of integrality of the general system of social security

in health and catastrophic diseases in colombia

pues se trata de personas que requieren una atención mayor por la enfermedad catastrófica que padecen.

(iii) El principio de integralidad como una característica del sistema de salud (p.22)

El principio de integralidad según la corte constitucional parte de dos factores, el primero es de acuerdo a las necesidades que tenga el paciente, que va desde la educación, prevención hasta llegar a la rehabilitación $\mathrm{y}$ el segundo que estas necesidades se brinden de manera adecuada, pues no basta con que existan planes de salud establecidos y que no se garanticen de manera integral.

(iv) El principio de integralidad como una obligación de las condiciones en las que debe prestarse el servicio de salud (p.23)

El principio de integralidad ha generado la obligación a los jueces de tutela de ordenar a la prestadora de salud que garantice todos los servicios que necesite el paciente para así evitar que presenten una nueva tutela cada vez que requieran una prestación de salud, ocasionando que los jueces emitan en muchas ocasiones los llamados "fallos integrales" mediante los cuales no solo se ordenan los tratamientos que están solicitando los afiliados, sino también aquellos que en el futuro prescriba el médico tratante, estos fallos se basan en la facultad que tiene el juez para fallar extra y ultra petita cuando hay una vulneración de los derechos fundamentales.

Por otra parte, las personas que padecen enfermedades ruinosas o catastróficas en ocasiones el acceso a la salud es restringido, pues no solo tienen barreras económicas sino geográficas y culturales. En cuanto a las barreras geográficas se hace referencia a los largos trayectos que deben de recorrer los pacientes para asistir a una cita médica o adquirir un medicamento o tratamiento, y más aquellos que residen en la zona rural, pues veces no tienen ni siquiera medio de transporte para llegar donde su prestadora de salud, generando que en muchos momentos tengan que cargar con consecuencias irremediables. Frente a las fronteras culturales consiste en la poca información que brindan los prestadores de salud con respecto a los derechos de los afiliados, desde la etapa de la prevención y educación hasta la fase de rehabilitación y finalmente se hace referencia a las barreras económicas cuando el afiliado no cuenta con capacidad económica para poder sufragar todos los tratamientos que requiere, pues en algunas ocasiones la EPS no brinda un servicio integral generando que sea el afiliado el que asuma dicha carga.

\section{LAS ENFERMEDADES CATASTRÓFICAS O RUINOSAS}

Según Quintero y Correa (2009), la cobertura de las enfermedades catastróficas o de alto costo, antes de la expedición de la Ley 100 de 1993 se limitaba solo a los afiliados a la seguridad social, generando que no existiera una mayor protección hacia las personas que padecían dichas enfermedades. Posteriormente, se dio mayor reconocimiento a estas enfermedades a partir de la expedición de la Resolución 5261 de 1994 más exactamente en el artículo 16: "Para efectos del presente decreto se definen como enfermedades ruinosas o catastróficas, aquellas que representan una alta complejidad técnica en su manejo, alto costo, baja ocurrencia y bajo costo efectividad en su tratamiento" Y en el artículo 17 se estableció el listado de tratamientos para las enfermedades ruinosas o catastróficas, definidas así:

Para efectos del presente Manual se definen como aquellos tratamientos utilizados en el manejo de enfermedades ruinosas o catastróficas que se caracterizan por un bajo costo- efectividad en la modificación del pronóstico y representan un alto costo.

Se incluyen los siguientes:

a. Tratamiento con radioterapia y quimioterapia para el cáncer.

b. Diálisis para insuficiencia renal crónica, trasplante renal, de corazón, de medula ósea y de córnea.

c. Tratamiento para el SIDA y sus complicaciones.

d. Tratamiento quirúrgico para enfermedades del corazón y del sistema nervioso central.

e. Tratamiento quirúrgico para enfermedades de origen genético o congénito.

f. Tratamiento médico quirúrgico para el trauma mayor.

g. Terapia en unidad de cuidados intensivos.

h. Reemplazos articulares. 
PARÁGRAFO. Los tratamientos descritos serán cubiertos por algún mecanismo de aseguramiento y estarán sujetos a períodos mínimos de cotización exceptuando la atención inicial y estabilización del paciente urgente, y su manejo deberá ceñirse a las Guías de Atención Integral definidas para ello.

Las enfermedades catastróficas generan un gran impacto en las personas que las padecen, ya que afecta muchas áreas de su vida, como su condición física, socioeconómica y psicológica, esta última se debe a que las enfermedades catastróficas también se acompañan de una desorganización psicológica y social generando obstáculos para el proceso de recuperación y rehabilitación. En Colombia a través de la Ley 100 de 1993 se establece que se debe seguir una guía de atención integral para la cobertura de las enfermedades catastróficas, considerado como el "... conjunto de actividades y procedimientos más indicados para el abordaje de la promoción y el fomento de la salud, la prevención, el diagnóstico, el tratamiento y la rehabilitación". En muchos países del mundo incluido Colombia, los trastornos médicospsicológicos asociados a las enfermedades catastróficas son evidentes, pero no existe un tratamiento apropiado que ayude a combatirlo, generando así que no se realice un control sobre el impacto que puede tener en la rehabilitación de las personas (Rincón, 2006). En este sentido Lopera afirma que:

[...] las enfermedades son complicadas desde el punto de vista clínico. La mayoría son crónicas y progresivas, pueden ser discapacitantes o dejar secuelas residuales, en especial en ausencia de programas de control eficaces. Lo paradójico es que muchas de estas enfermedades son prevenibles, pero cuando se presentan demandan atención prolongada, continua, costosa y de alta complejidad. (p. 123)

En cuanto al impacto económico, estas enfermedades no solo incapacitan o matan a las personas también genera un detrimento económico tanto a los que las padecen como a sus familias, por ello si el paciente no recibe un tratamiento adecuado, se le condena a un deterioro progresivo de su salud y calidad de vida, por eso estas enfermedades no sólo generan un problema médico, sino que se configura un desafío económico y social. No se trata de enfermedades comunes sino de afecciones que son difíciles por los siguientes aspectos: a) El clínico, porque en muchas ocasiones hay incertidumbre sobre las modalidades de abordaje.

b) El económico, porque los importantes costos que involucran su diagnóstico y atención comprometen la sostenibilidad de los tratamientos y repercuten en gran manera sobre las finanzas de quienes deben pagar por ellos (los pacientes, sus familias o los sistemas de salud).

c) El ético, porque la diseminación del uso de nuevas tecnologías terapéuticas puede resultar más acelerada que la generación de evidencias confiables sobre su seguridad y beneficios terapéuticos, lo que a menudo convierte al paciente en un conejillo de indias sobre el cual se ponen a prueba tratamientos sin la evidencia científica necesaria.

d) El distributivo, porque cuando los sistemas de salud se hacen cargo de financiar los tratamientos, concentran una gran parte de sus recursos sobre unos pocos pacientes que, desafortunadamente, tienen poca o ninguna probabilidad de sanar. En contrapartida, existen muchos tratamientos de comprobada efectividad que pueden ser utilizados para combatir enfermedades más simples, pero que aún no fueron implementados en todo su potencial, sobre todo en los países en desarrollo. Por lo tanto, si esos recursos que se están destinando a las enfermedades más caras se emplearan para extender la cobertura y mejorar la respuesta que se da a las enfermedades más simples, probablemente se obtendrían mejores resultados sanitarios para un mayor porcentaje de la población. En otras palabras, con la misma inversión dinero, se compraría más salud. (Burgin, 2014 p.14)

En el artículo 16 de la Resolución 5261 de 1994 se define las enfermedades catastróficas "como aquellas que representan una alta complejidad técnica en su manejo, alto costo, baja ocurrencia y bajo costo-efectividad en su tratamiento". Hasta ahora, existe una lista de estas enfermedades que son reguladas en la mencionada resolución, se consideran eventos de alto costo las siguientes enfermedades:

[...] la enfermedad renal crónica en estadío 5 (ERC-5), que implica procedimientos PTAC como la diálisis y trasplante renal, la enfermedad cardiovascular (ECV), que amerita tratamientos quirúrgicos o trasplante de corazón, otras enfermedades que requieren trasplantes (médula ósea y córnea), algunos tipos de cáncer que implican tratamientos de 
salud y las enfermedades catastróficas en colombia

The principle of integrality of the general system of social security

in health and catastrophic diseases in colombia

radioterapia y quimioterapia, los tratamientos antirretrovirales y el manejo de complicaciones para el sida, reemplazos articulares, tratamientos quirúrgicos para enfermedades del sistema nervioso central, enfermedades genéticas o congénitas, el trauma mayor y la terapia en unidad de cuidados intensivos (7). La norma clasifica la atención por consulta externa, hospitalización y urgencias como de alto costo, si es compatible con los eventos o tratamientos definidos normativamente, y como consultas por enfermedad general cuando un paciente con EAC utiliza los servicios por causas que pueden o no estar asociadas con la enfermedad de base. (p.123)

Infortunadamente, los recursos destinados para el cubrimiento de patologías definidas como catastróficas no son suficientes para lograr una atención eficiente en los pacientes, además no existe mayor intervención, vigilancia y control por parte de los entes reguladores, los cuales han consentido que las entidades prestadoras de salud impongan restricciones al acceso a la salud, lo cual va en contra de los principios establecidos en la Ley 100 de 1993, entre los que se encuentra el principio de integralidad (Gil y Camargo, 2006). Las enfermedades catastróficas afectan la parte física, psicológica y económica tanto de la persona que padece la enfermedad como la de su familia, generando con ello, que no se de una protección integral.

\section{ANÁLISIS JURISPRUDENCIAL DE LA RELACIÓN ENTRE EL PRINCIPIO DE INTEGRALIDAD Y LAS ENFERMEDADES CATASTRÓFICAS}

\section{Sentencia T-894 de 2013: la epilepsia}

En la Sentencia T-894 de 2013, se analiza la enfermedad de epilepsia, y la exoneración de copagos para las personas que padecen enfermedades catastróficas con base en los siguientes hechos:

La accionante manifiesta que su hijo en diciembre de 2007 sufrió un ataque convulsivo, generando que necesitará varios exámenes para su diagnóstico. Estos procedimientos fueron realizados por la Nueva EPS, en cumplimiento del fallo de tutela. Una vez realizado el procedimiento fue diagnosticado con "epilepsia partialis continua (Kojewnikow) y encefalitis de Rasmussen" (párr.19). Consideradas como enfermedades catastróficas. A finales del mes de Noviembre de 2008 a Dairo Fernando Oliveros Cárdenas le fue realizado "una hemisferectomía funcional, que es la extracción o inhabilitación de un hemisferio cerebral" (párr.20). Posterior a este procedimiento, el menor tuvo que someterse a terapias de rehabilitación. Sin embargo, manifiesta la agente oficiosa que los copagos de dichos servicios se han convertido en una barrera para la rehabilitación de su hijo ya que carece de capacidad de económica para sufragar los gastos que se genere por la autorización, además manifiesta que es madre cabeza de hogar y lo ingresos que tiene son para sufragar todos los gastos que requiere la casa y su hijo, por lo cual solicita que se exonere del pago de cuotas moderadoras y copagos por los servicios que necesite su hijo.

En primera instancia, el Juzgado Sexto de Ejecución de Penas y Medidas de Seguridad de Cali en providencia del 18 de abril de 2013 , decidió acceder a las pretensiones realizadas por la señora Yamile Cárdenas, puesto que al estar el menor en situación de debilidad la falto del pago de copagos o cuotas moderadoras genera que no se pueda continuar con las terapias que debe recibir Dairo Fernando Oliveros Cárdenas.

La entidad demanda impugnó la decisión, por lo cual, en segunda instancia conoció del proceso el Tribunal Superior de Cali, Sala de Decisión Constitucional, en donde a través de la sentencia del 9 de julio revocó el fallo del a quo, argumentando que el Juez de primera Instancia debía haber resuelto el problema a través de incidente de desacato frente al fallo del 2008, en donde se había ordenado a la Nueva EPS que garantizara un tratamiento integral.

El planteamiento jurídico que estableció la Corte Constitucional fue

[...] ¿desconoce el derecho fundamental a la salud y a la vida digna la negativa de una EPS del régimen contributivo a exonerar del cobro de pagos moderadores a tres pacientes con epilepsia $\mathrm{y}$ otros trastornos neurológicos, teniendo en cuenta que el núcleo familiar asegura no tener recursos suficientes para sufragar los mismos? (párr.87)

Para dar solución al problema planteado la Corte analizó que la epilepsia está incluida dentro de las enfermedades descritas en el artículo $1^{\circ}$ de la Resolución 3974 de 2009 como catastróficas o de alto costo, las cuales están exentas del pago de copagos en su tratamiento de acuerdo con el artículo $7^{\circ}$ del 
Acuerdo 260 de 2004. También se llega a la misma conclusión con referencia a las cuotas moderadoras, puesto que en el parágrafo $2^{\circ} \mathrm{del}$ artículo 6 del Acuerdo 260 de 2004,

[...] se estableció que, si el usuario está inscrito o se somete a las prescripciones regulares de un programa especial de atención integral para patologías específicas, en el cual dicho usuario debe seguir un plan rutinario de actividades de control, no habrá lugar a cobro de cuotas moderadoras en dichos servicios. (párr.132).

Finalmente, la Corte ordenó revocar la sentencia de tutela de segunda instancia y ordenó a la Nueva EPS de no solicitar pagos de copagos o cuotas moderadoras para brindarle los servicios de salud al accionante.

En este caso, se logra ver la importancia de la aplicación del principio de integralidad, puesto que a través de él se puede garantizar que las personas puedan tener una rehabilitación óptima, además se debe de brindar todos los tratamientos que conlleven al mejoramiento de su salud y calidad de vida. En la situación que es objeto de análisis se logra ver que por la EPS establecer copagos y cuotas moderadoras que los usuarios no tienen capacidad de sufragar se vulnera el derecho fundamental a la salud, debido a las barreras económicas que se originan.

\section{Sentencia T-499 de 2014: el cáncer}

En la Sentencia T-499 de 2014, se estudia la enfermedad catastrófica "cáncer" con base en el principio de integralidad y haciendo referencia a los siguientes hechos:

La señora Flor María Rivera Rojas, quien actúa como agente oficiosa de su hermana Yolanda Rivera Rojas, presenta acción de tutela contra Cafesalud E.P.S, en razón a que la señora Yolanda Rivera Rojas tiene 53 años de edad, y a mediados del año 2013 le diagnosticaron Cáncer de pleura metastásico desconocido, por lo cual el médico de la EPS Cafesalud le ordenó una "tomografía axial computada" (TAC), sin embargo este examen nunca se llevó a cabo, ya que la EPS nunca expidió la autorización correspondiente, por tanto ante dicha omisión, la agente oficioso acudió ante el Instituto Nacional de Cancerología para que le realizaran los exámenes prescritos, en donde tenía que asumir todos los costos, luego, los resultados se llevaron a Cafesalud, quien autorizó una biopsia que no dio resultado alguno ya que la muestra extraída era demasiado pequeña, generando con ello una demora injustificada del tratamiento por parte de la EPS, por lo cual se volvió acudir al Instituto Nacional de Cancerología, en donde se prescribió la práctica de los siguientes exámenes, «cita $x$ cuidados paliativos y dolor, tac de abdomen y pelvis, tac de tórax, broncoscopia fibro-óptica con lavado bronquial, broncoscopia fibro-óptica con función trasntraqueal o trasnsbronquial con aguja, estudio de coloración básica en citología, estudio de coloración inmunohistoquímica en biopsia, estudio de coloración básica en biopsia, cita $x$ neumología» (párr.34)

Frente a la anterior prescripción médica emitida por el Instituto Nacional de Cancerología, la EPS Cafesalud no autorizó la totalidad de los procedimientos aduciendo que algunos de dichos exámenes no eran importantes para el estado del paciente de acuerdo a lo dicho por el médico de la EPS y además que algunos de ellos no hacían parte del Plan Obligatorio de Salud, como son la "Gamagrafía Osea y Filtración Glomerular". De acuerdo a esta respuesta, la agente oficiosa solicitó la remisión al Instituto Nacional de Cancerología, sin embargo, la EPS respondió que no tenía convenio vigente con el instituto. Además, manifestó que la enfermedad que tenía su hermana necesita de un tratamiento integral y rápido la cual no ha sido prestado por la EPS Cafesalud, colocando en riesgo la salud de la señora Yolanda Rivera Rojas, por lo cual sus pretensiones es que la autorización y práctica de los tratamientos prescritos por el Instituto Nacional de Cancerología ESE se realice en el mismo instituto, como también que se garantice un tratamiento integral.

La sentencia de primera instancia fue resuelta por el Juzgado Cuarenta y Seis Penal Municipal con Función de Control de Garantías, a través de providencia del 16 de diciembre de 2013 negó la acción de tutela aduciendo que los servicios fueron suministrados por el médico tratante y además autorizaron los exámenes de "GAMAGRAFIA DE FILTRACIÓN GLOMERULAR y GAMAGRAFIA OSEA" (párr.36). En cuanto al tratamiento integral la EPS Cafesalud ya autorizó todo lo solicitado por la accionante por lo cual no hay lugar para que se le concedan las peticiones a la tutelante.

La decisión fue impugnada por la señora Yolanda Rivera Ríos y mediante sentencia del 31 de enero de 2014, el Juzgado Veinticinco Penal del Circuito con Función de Conocimiento de Bogotá confirmó el fallo proferido por el a quo. El problema jurídico que 
salud y las enfermedades catastróficas en colombia

The principle of integrality of the general system of social security

in health and catastrophic diseases in colombia

entra a resolver la Corte Constitucional es si la negativa de los servicios de salud por parte de la EPS Cafesalud que luego fueron autorizados cuando se instauró la tutela, genera una mala prestación del servicio de salud que otorga el cambio de la actual IPS al Instituto Nacional de Cancerología, aun cuando no haga parte de su red de instituciones prestadoras de salud.

Con respecto a este problema jurídico la Corte analiza varios aspectos, entre los cuales dice que por regla general el médico que se encuentra adscrito a la EPS es el que le puede prescribir un servicio a un paciente, sin embargo, cuando un médico no adscrito a la EPS emite un concepto no debe de ser rechazado de manera instantánea bajo el supuesto de que no se encuentra adscrito a la EPS, ya que este puede ser vinculante, además también esto conlleva a entender la problemática que se presenta en la libertad de escoger las IPS para que se garantice la atención en la salud, ya que la Entidad Promotora de Salud es la encargada de brindar a todos sus afiliados las instituciones que presten tratamientos de manera efectiva, de esta forma los afiliados pueden elegir libremente entre las IPS que brinda las EPS, sin embargo, existe una excepción para acceder a aquellas IPS que no tengan convenio, y es que solo se puede dar cuando las IPS que otorga las EPS, no cuenten con la capacidad o teniéndola no brinden la prestación de manera efectiva, generando así la vulneración de sus derechos fundamentales.

En este caso, tanto la EPS como las IPS no han brindado el tratamiento integral que el paciente necesita debido a la enfermedad que padece, ya que los servicios que se han brindado han sido lentos e ineficaces para combatir la enfermedad generando así la evolución del cáncer en el cuerpo de la accionante, un ejemplo de ello, es que el médico tratante de la EPS ordenó "una Tomografía Axial Computada (TAC)", la cual nunca fue practicada, generando que se tuviera que hacer de manera particular. De esta forma, la Corte concluye que la EPS Cafesalud no cuenta con IPS idóneas para garantizar el servicio integral a la accionante, por lo tanto, decide revocar el fallo de primera instancia y conceder el amparo de los derechos fundamentales a la salud, la vida y la integridad personal.

\section{Sentencia T-421 de 2015: la insuficiencia renal crónica}

En la Sentencia T-421 de 2015, se estudia la "Insuficiencia Renal Crónica", catalogada como una enfermedad catastrófica, con base en los siguientes hechos:

\begin{abstract}
El señor Federico de los Santos Plaza Velázquez, de 59 años de edad, presentó un derecho de petición ante Coomeva EPS para que le dieran las autorizaciones de varios medicamentos $\mathrm{y}$ exámenes clínicos pre quirúrgicos que fueron ordenados por sus médicos tratantes en razón a su diagnóstico el cual fue falla renal terminal y retinopatía diabética. Debido a esta enfermedad, el requiere controles periódicos con especialistas, y necesita medicamentos para poder combatir su enfermedad, sin embargo, la EPS Coomeva le da respuestas evasivas, aun sabiendo que es una persona de la tercera edad que padece una enfermedad catastrófica.
\end{abstract}

El accionante radico derecho de petición, en donde solicitaba que padecía diabetes tipo 2 , y que por tanto requería hemodiálisis tres veces por semana, además esta patología derivó en un diagnóstico de "Retinopatía Diabética Proliferativa y Edema Macular Severo, por lo tanto, su oftalmólogo lo remitió al retinólogo, quien le formuló un medicamento (Ranibizumab de $10 \mathrm{mg} / \mathrm{ml}$, presentación ampolla $\mathrm{N}^{\mathrm{o}} 2$, uso hospitalario medicamento intravítreo para ambos ojos)" (párr.20). Pero la EPS Coomeva solo le entregó este medicamento un año después de que lo solicitó, generando perjuicios irremediables frente al accionante, ya que la falta de medicamento deterioró su visión, por lo cual necesitó otra valoración por un médico especialista, a pesar que el retinólogo dio "varias órdenes de exámenes y una cirugía urgente de aspiración diagnóstica con vítreos, honorarios de anestesia, terapia antiangiogénica con lucentis ojo derecho" (párr.21). La EPS retrasó la autorización de estos procedimientos, generando que esté en riesgo la visión del accionante. El 09 de Julio de 2014, el accionante asistió a una cita en la Fundación Cardioinfantil de Bogotá, en donde inició un estudio de pre trasplante renal. Después, de haberse realizado los correspondientes exámenes, la junta médica autorizó el trasplante, por lo que se vio en la obligación de acudir ante Coomeva EPS con el objetivo que le dieran autorizaciones que 
requería para ser incluido en lista de espera, sin embargo, la EPS le respondió que no pueden ser autorizadas porque las fórmulas son ilegibles y no muestran el nombre de su médico.

La decisión de primera instancia fue emitida por el Juzgado Primero Promiscuo Municipal de Maicao a través de sentencia del veintinueve (29) de diciembre de dos mil catorce (2014), en donde le ordenó a Coomeva EPS dar respuesta escrita y de fondo del derecho de petición en donde se solicitó todos los procedimientos y medicamentos, sin embargo, no ordenó que se autorizaran los medicamentos y exámenes clínicos solicitados por el accionante, porque adujo que esto debería de solucionarse a través de la respuesta del derecho de petición.

Este caso, fue seleccionado para revisión constitucional por la Sala de Selección de Tutelas Número Tres de esta corporación, en donde el magistrado adoptó las medidas provisionales que consideró necesarias para proteger los derechos fundamentales del accionante y por ende ordenó que la EPS Coomeva le debía brindar una atención integral para la atención de sus patologías. El problema jurídico que se suscita es determinar si Coomeva EPS vulneró el derecho de petición del accionante por no responder el derecho de petición en donde se solicitaba las prestaciones prescritas por sus médicos tratantes, y si por esta conducta se comprometió el derecho fundamental a la salud del accionante.

En la Resolución 5261 de 1994 en su artículo 16 "se define las enfermedades catastróficas como aquellas que representan una alta complejidad técnica en su manejo, alto costo, baja ocurrencia y bajo costo-efectividad en su tratamiento". En dicha resolución se caracterizó a la insuficiencia renal crónica como una enfermedad catastrófica o ruinosa. En este pronunciamiento, la Corte Constitucional muestra un panorama de obstáculos los cuales se ven enfrentados los pacientes que padecen esta enfermedad renal crónica para acceder a los servicios de salud:

“i) El retraso o la negativa a entregar las autorizaciones necesarias para que los pacientes de enfermedad renal crónica se sometan a un trasplante de riñón" (párr.106).

Existen muchas tutelas que han sido instauradas por pacientes de enfermedad renal crónica con la finalidad de que su EPS puedan brindarles sus tratamientos, medicamentos, procedimientos que requieren para lograr restablecer su salud, inclusive en muchas ocasiones, los pacientes que están en etapa terminal la EPS suspenden sus tratamientos o no hay oportunidades para salir en la lista de candidatos de trasplante de riñón, en razón a las barreras administrativas a las que son sometidas.

“ii) El retraso o la negativa a autorizar los servicios, medicamentos, insumos y procedimientos que demanda la atención de la enfermedad renal crónica" (párr.115).

Existen muchos fallos que se han encargado de amparar el derecho fundamental a la salud, en algunos se ha ordenado que se practiquen todos los servicios que requiera el paciente, como pañales o medicamentos, pues estos insumos no suelen otorgarlo las EPS, sustentándose en que no se encuentran dentro del plan de beneficios.

“iii) La renuencia a cubrir los gastos de transporte del paciente hasta el lugar donde se realizan las terapias e intervenciones que requiere para el manejo de la enfermedad renal crónica" (párr.126).

Las personas que padecen insuficiencia renal crónica tienen muchas barreras para acceder al servicio de salud de manera oportuna, pues uno de los mayores obstáculos que tienen son por el medio de transporte, ya que estos pacientes necesitan realizar terapias de diálisis varias veces a la semana, pero no cuentan ni con el medio de transporte ni con capacidad económica para sufragarlo y las EPS no se lo garantizan. Estas barreras tienen un impacto mayor en las personas que residen en zonas como el chocó, putumayo y Amazonas, donde los centros clínicos especializados son casi nulos y no cuentan con políticas de protección para las personas que padecen estas enfermedades ruinosas.

“iv) La interrupción injustificada del tratamiento, por cuenta de un cambio en las condiciones de prestación del servicio: el traslado de IPS, por decisión de las empresas prestadoras" (párr.133).

En el caso que no se tenga convenio con la IPS elegida por el afiliado, es deber de su EPS de garantizar un tratamiento completo y oportuno, pues el paciente no puede soportar que por cuestiones administrativas su servicio no se brinde de manera adecuada y eficiente, ya que se está frente a una enfermedad muy grave que requiere que los servicios se presten con la mejor calidad. En el caso particular, como la EPS Coomeva no autorizó oportunamente las prestaciones que requería el accionante y al retrasar la autorización de los medicamentos y procedimientos ordenados por su retinólogo su vista empeoró, reflejando el incumplimiento de la Eps de todos los presupuestos legales y 
salud y las enfermedades catastróficas en colombia

The principle of integrality of the general system of social security

in health and catastrophic diseases in colombia

jurisprudenciales mencionados anteriormente, por lo que la Corte decide revocar la sentencia proferida por el Juzgado Primero Promiscuo Municipal de Maicao y ampara el derecho fundamental a la salud del peticionario.

\section{Sentencia T-081 de 2016: el cáncer}

En la Sentencia T-081 de 2016, se analiza el cáncer como enfermedad catastrófica basada en los siguientes hechos:

La señora Isabel Guevara Millán de 62 años de edad, padece cáncer de seno, no cuenta con capacidad económica y tiene a su cargo a dos menores de edad. El 15 de Julio de 2015, el médico tratante, le formuló unos medicamentos, sin embargo, la EPS-s Caprecom no autorizó ni entregó los medicamentos prescritos, lo único que hizo fue dilatar la prestación del servicio, por cual la accionante solicitó que sean protegidos sus derechos fundamentales a la salud y a la vida, y que se haga entrega de dichos medicamentos.

En primera instancia conoció el Juzgado Séptimo Civil del Circuito de Bogotá, quien mediante providencia del 20 de agosto de 2015, negó las pretensiones, aduciendo que la señora Isabel Guevara Millán no anexó la orden médica de los medicamentos, además, manifestó que el médico tratante pertenece al Instituto Nacional de Cancerología, entidad con la cual Caprecom EPS-S no tiene ningún vínculo. Frente a esta decisión no se interpuso recurso alguno, sin embargo, si pasó a la Sala Cuarta de Revisión.

El problema jurídico que se suscita es determinar si Caprecom EPS-S vulneró los derechos fundamentales a la salud y a la vida de la señora Isabel Guevara Millán porque no autorizó los medicamentos que ella requería por su enfermedad catastrófica que padecía.

El tratamiento integral está regulado en el artículo 8 de la Ley 1751 de 2015 donde establece que "el servicio de salud debe incluir todos aquellos medicamentos, exámenes, procedimientos, intervenciones y terapias, entre otros, con miras a la recuperación e integración social del paciente, sin que medie obstáculo alguno independientemente de que se encuentren en el POS o no". Igualmente, comprende un tratamiento sin fracciones. En el caso de las personas que padecen cáncer, la cual es una enfermedad catastrófica requiere un tratamiento continuo que no puede sujetarse a dilaciones injustificadas ni prestarse de forma incompleta, se debe de tener en cuenta que las personas que padecen estas enfermedades no tienen la misma capacidad que tienen las demás para hacer valer sus derechos, por lo tanto, se les debe brindar un servicio eficiente desde el inicio de la enfermedad hasta llegar a la rehabilitación, ya que cuando los trámites son excesivos e injustificados generan que el tiempo de sufrimiento se extienda por las dolencias del paciente, generando en muchas ocasiones la muerte.

En el caso concreto, a pesar que no exista una orden médica, lo que en realidad importa y que no se debe de discutir es que la accionante padece cáncer, por tanto, tiene una enfermedad para la que es obligatorio ordenar un tratamiento integral. Por lo anterior, la Corte decide revocar el fallo proferido, el 20 de agosto de 2015, por el Juzgado Séptimo Civil del Circuito de Bogotá y en su lugar se tuteló los derechos fundamentales salud y a la vida de la señora Isabel Guevara Millán.

En la sentencia T-020 del 2017, se indicó que la salud es un derecho fundamental que se define como "la facultad que tiene todo ser humano de mantener la normalidad orgánica funcional, tanto fisica como en el plano de la operatividad mental, y de restablecerse cuando se presente una perturbación en la estabilidad orgánica y funcional de su ser" (párr.4).

De lo anterior, para materializar el derecho a la salud se debe brindar de manera integral el servicio de salud, en donde incluye un diagnóstico efectivo, que lleva consigo lo siguiente: “(i) una valoración oportuna sobre las dolencias que aquejan al paciente, (ii) la determinación de la enfermedad que padece $y$ (iii) el establecimiento de un procedimiento médico específico a seguir para lograr el restablecimiento de su salud" (párr.8).

Conforme a lo anterior, el derecho a un diagnóstico efectivo se puede ver trasgredido cuando las EPS no permiten o se demoran en realizar el diagnóstico y la prescripción efectiva de un tratamiento para remediar una enfermedad, cuando esto sucede, la Corte ha dicho que el paciente está en la plena capacidad de exigir a la EPS y a su médico tratante emitir un diagnóstico y una prescripción de manera que permita iniciar un tratamiento médico encaminado a aliviar sus padecimientos. 
En conclusión, el derecho fundamental a la salud, se debe prestar bajo el principio de integralidad, ya que la salud es la facultad del ser humano de tener una normalidad orgánica, funcional y mental, por eso es que se le debe de garantizar a las personas su salud en condiciones dignas, ya que es fundamental para el ejercicio de otros derechos fundamentales

\section{Sentencia T-387 de 2018: el cáncer de lengua}

En la Sentencia T-387 de 2018, se analiza el cáncer de lengua, catalogado como una enfermedad catastrófica, con base en los siguientes hechos:

La señora, Sor Adiela Sánchez Quintero, en calidad de agente oficiosa de su hermano Adalberto Antonio Sánchez Quintero, instauró acción de tutela contra CoomevaEPS(Medellín) en razón a que la entidad no ha suministrado de forma diligente la prestación de los servicios de salud de forma integral porque no existe una orden medica que lo fundamente. Frente a esto, la Corte Constitucional manifiesta que las personas que padecen enfermedades catastróficas o ruinosas, como el cáncer, tienen derecho a una protección reforzada por parte del Estado, la cual consiste en otorgarles un acceso sin barreras y un tratamiento integral oportuno, independiente de que se encuentren en el Plan Obligatorio de salud o no. Lo anterior permite entender que la integralidad no solo comprende el conjunto de medicamentos, exámenes, tratamiento y todo lo que requiera el paciente para restablecer su salud, sino que también la integralidad comprende la garantía de recibir servicios de apoyo social como en el área psicología, laboral, familiar y social. Además, el servicio de salud no debe de ir encaminado a solo superar las afecciones que perturban las condiciones físicas o mentales del paciente, sino también a sobrellevar la enfermedad garantizando su integridad personal, es decir, debe de estar dirigido a que su entorno sea tolerable y digno, puesto que para que se ampare el derecho fundamental a la salud no se necesita que el paciente este en una situación determinada que amenace su vida de forma grave, sino que simplemente que aquel se encuentre en condiciones indignas de existencia, como es que no puedan soportar intensos dolores.

\section{Sentencia T-010 de 20: tumor benigno}

En la Sentencia T-010 de 2019, se analiza el principio de integralidad a través del caso de la señora Sandra Liliana Villareal López, actuando en representación de su menor hija, Laura Daniela Abril Villareal, se instauró acción de tutela contra la NUEVA E.P.S por vulnerar sus derechos fundamentales a la salud, a la vida digna, a la igualdad, y la integridad física, pues esta entidad le negó a la menor una intervención quirúrgica de "resección de tumor benigno en el lóbulo de la oreja" derecha ordenada por el médico tratante, aduciendo que tenía una finalidad estética y no funcional. Frente a lo anterior, la Corte Constitucional ha expresado que el principio de integralidad está regulado entre otras en la Ley 1122 de 2007 y Ley 1751 de 2015, por vía jurisprudencial a través de la sentencia C-313 del 2014, se permitió que las exclusiones en el servicio de salud que establecidas en la ley estatuaria pueden ser autorizadas siempre que cumplan con los requisitos de exigidos para garantizar la integralidad en el servicio. Finalmente, el principio de integralidad obliga al Estado y a las entidades encargadas de brindar el servicio de salud, a garantizar la autorización completa de todos los procedimientos que requiera el paciente.

\section{Sentencia T-062 del 2019: "nefropatia IgA Proliferativa Mesanguial", insuficiencia renal crónica}

En la sentencia T-062 del 2019, se estudia el caso del señor Carlos Emilio Mosquera Cicero, quien fue diagnosticado con una enfermedad progresiva, catastrófica y terminal, denominada: "nefropatía $\operatorname{Ig} A$ Proliferativa Mesanguial, Insuficiencia Renal Crónica”, el cual solicitaba a su EPS Establecimiento de Sanidad Militar 500018403182 -de la Fuerza Aérea- el traslado al Hospital Universitario Hernando Moncaleano Perdomo de la ciudad de Neiva para que le realizaran un trasplante de órganos, pues a pesar que ya había sido incluido en la lista de espera de donantes en la ciudad de Bogotá, el afiliado, consideraba que tenía mayor oportunidades para tener el trasplante en la ciudad Neiva, en razón a que el número de pacientes en espera en Bogotá son mayores que en Neiva, generando que la espera pueda incluso durar años. Asimismo, el Hospital Universitario Hernando Moncaleano Perdomo de la ciudad de Neiva, contaba con 
salud y las enfermedades catastróficas en colombia

The principle of integrality of the general system of social security

in health and catastrophic diseases in colombia

todos los elementos para realizar el trasplante y tratar su patología de manera integral.

Sin embargo, la Eps negó esta solicitud argumentando que no tenía convenio con la mencionada IPS, a pesar que el afiliado pudo probar que la EPS si contaba con dicho convenio. Por lo anterior, el señor Mosquera Cicero presentó acción de tutela por la vulneración de sus derechos fundamentales a la salud, a la seguridad social, a la vida en condiciones dignas y a la igualdad por parte de la entidad en mención.

En primera instancia, el juzgado $1^{\circ}$ Civil del Circuito de Neiva negó la acción de tutela aduciendo que así se realice el traslado a una regional diferente y se cambie de IPS, eso no le da seguridad de que se haga de inmediato el trasplante, además que, al estar inscrito en Bogotá, tiene más oportunidades de recibir el trasplante, pues se recibe donaciones de todo el país.

El Tribunal Superior del Distrito Judicial de Neiva, Sala Tercera de Decisión Civil Familia Laboral, revocó la decisión de primera instancia, basándose en que los afiliados a las entidades administradoras de los servicios de salud pueden elegir las IPS donde se lleven a cabo los tratamientos que requiera, siempre que se encuentren en la red de servicios contratada.

Por lo anterior, la Corte Constitucional en sede de revisión, consideró que con base en el derecho a la salud el usuario puede elegir la IPS que brinde sus tratamientos, siempre que tenga convenio con la EPS a la cual está afiliado. En el caso de personas con enfermedades catastróficas que necesiten trasplante de órganos, pasa exactamente lo mismo, estas personas pueden trasladarse a la IPS que elijan, y que se encuentren dentro de la red de servicios de la EPS, pues el derecho a la libre escogencia facultad al afiliado que en caso que requiera una IPS que se encuentra en una regional diferente a la que se encuentra, pueda solicitar el traslado, con el fin de obtener protección de su derecho fundamental a la salud.

\section{CONCLUSIÓN}

El principio de integralidad se encuentra establecido en el artículo 2 de la Ley 100 de 1993 "como la cobertura de todas las contingencias que afectan la salud, la capacidad económica y en general las condiciones de vida de toda la población". A la luz de la Corte Constitucional este principio comprende el "suministro de medicamentos, intervenciones quirúrgicas, prácticas de rehabilitación, exámenes para el diagnóstico y el seguimiento, que inicia desde la prevención hasta llegar a la rehabilitación del paciente". La aplicación del principio de integralidad es fundamental para las personas que padecen enfermedades catastróficas, pues cubre todas las áreas del bienestar humano.

En la actualidad, las personas que padecen dichas enfermedades se encuentran con muchas barreras para acceder al sistema de salud, debido a la falta de diagnóstico efectivo y la negación de medicamentos. La Corte Constitucional en reiteradas sentencias establece el principio de integralidad como parte fundamental para garantizar el derecho a la salud, no obstante, la solución no radica en que se tengan que acudir a la acción de tutela por cada servicio que se niega, el sistema general de seguridad social a través de sus órganos debe garantizar este principio, para que tenga mayor relevancia y aplicación en el sistema de salud.

Otro problema, es la falta de información al desconocer que la enfermedad es catalogada como catastrófica, en la mayoría de los casos las EPS aun conociendo este tipo de patologías, hacen caso omiso y los pacientes muchas veces no tienen acceso a los tratamientos porque son costosos, sin saber que por padecer este tipo de enfermedades están exentos de sufragar copagos, y tienen derecho a recibir una atención integral, en estos eventos es importante que las enfermedades consideradas como catastróficas tengan un mayor desarrollo, deben ser más específicas en el sentido de explicar sus tratamientos y las EPS deben conocer todo lo concerniente, para no trasgredir derechos fundamentales con las barreras que existen para acceder a la atención de la salud.

Finalmente, aunque la normatividad colombiana establece en el artículo 16 de la Resolución 5261 de 1994 la baja ocurrencia de las enfermedades catastróficas, lo cierto es que genera un impacto negativo en la salud; solo la materialización del principio de integralidad garantiza que aquellos que padecen enfermedades ruinosas gocen de una atención oportuna, eficiente y con calidad. 


\section{BIBLIOGRAFÍA}

Arenas, G. (2008). El derecho colombiano de la seguridad social. Bogotá, Colombia: Legis

Garzón Landínez, T. (2014). El Plan Obligatorio de Salud en Colombia y su rol en el cumplimiento del Principio de Integralidad en Salud para las personas que padecen VIH. Universidad Nacional de Colombia, pp.1-64. http://bdigital.unal.edu.co/11853/1/ tarycuyanagarzonlandinez.2014.pdf

Lopera, M. (2017). Utilización de servicios de salud por enfermedades catastróficas o de alto costo en Antioquia. Revista Gerencia y Políticas de Salud, Volumen 16, pp.120-137. http://revistas.javeriana.edu.co/index.php/ gerepolsal/article/view/19891

Rocha, Buelvas, A. (2010). Antecedentes históricos de la seguridad social en salud: parte de la construcción de un país sin memoria. Revista CES, pp.67-70. https://dialnet.unirioja. es/servlet/articulo?codigo $=3705813$

Quintero Lyons, J. y Correa Solano, L. (2009). Enfermedades catastróficas en el sistema general de seguridad social en salud en Colombia.Revista Jurídica MarioAlarioD'Filippo.pp.10-27. http:// webcache.googleusercontent.com/search?q= cache:tQ77b6ezLWcJ:revistas.unicartagena. edu.co/index.php/marioalariodfilippo/ article/view/190/150+\&cd=6\&hl=es$419 \& \mathrm{ct}=\mathrm{clnk} \& \mathrm{gl}=\mathrm{co}$

Rincón et al.(2006).Atención psicosocial integral en enfermedad catastrófica (Atinar). Revista Colombiana de Psiquiatría, Volumen35, pp.44-71.http://www.scielo. org.co/scielo.php?script=sci_arttext\&pid $=$ S0034-74502006000500005

Burgin et al. (2014). Respuestas a las enfermedades catastróficas.https://www. cippec.org/wp-content/uploads/2017/03/1283. pdf

\section{Fuentes legales}

Constitucional nacional, articulo 48 y 49 .

Ley 90 de 1946.

Ley 10 de enero de 1990, articulo 4.
Ley 100 de 1993, artículo 2, 153, 156, 202 y 211.

Ley 972 DE 2005.

Ley 1151 de 2007.

Ley 1122 de 2007.

Ley 1751 de 2015, artículo 2, 8 y 15

Decreto 056 de 1975.

Decreto 1543 de 1997.

Resolución 5261 de 1994, articulo 16.

Resolución 3974 de 2009, articulo 1 y 2.

Resolución 5521 de 2013 artículo 126 y 137.

\section{Jurisprudencia}

Corte Constitucional de Colombia. Sentencia T-894 De 2013 (M.P.Jorge Iván Palacio Palacio: diciembre 3 del 2013).

Corte Constitucional de Colombia. Sentencia C- 313 de 2014 (M.P. Gabriel Eduardo Mendoza Martelo: mayo 29 de 2014).

Corte Constitucional De Colombia. Sentencia T-421 De 2015 (M.P. Myriam Ávila Roldán: julio 6 de 2015).

Corte Constitucional de Colombia. Sentencia T- 081 de 2016 (M.P. Gabriel Eduardo Mendoza Martelo: febrero 23 de 2016).

Corte Constitucional de Colombia. Sentencia T-020 de 2017 (M.P. Luis Ernesto Vargas Silva: enero 20 del 2017).

Corte Constitucional de Colombia. Sentencia T-387 de 2018 (M.P. Gloria Stella Ortiz Delgado: septiembre 21 del 2018).

Corte Constitucional de Colombia. Sentencia T-019 de 2019 (M.P. Cristina Pardo Schlesinger: enero 22 del 2019).

Corte Constitucional de Colombia. Sentencia T- 062 de 2019 (M.P. Alberto Rojas Ríos: febrero 18 del 2020) 